\title{
Effects of exercise on cognitive and motor function in chronic fatigue syndrome and depression
}

Susan K Blackwood, Siobhan M MacHale, Mick J Power, Guy M Goodwin, Stephen M Lawrie

\begin{abstract}
Objectives-Patients with chronic fatigue syndrome complain of physical and mental fatigue that is worsened by exertion. It was predicted that the cognitive and motor responses to vigorous exercise in patients with chronic fatigue syndrome would differ from those in depressed and healthy controls.

Methods-Ten patients with chronic fatigue syndrome, 10 with depressive illness, and 10 healthy controls completed cognitive and muscle strength testing before and after a treadmill exercise test. Measures of cardiovascular functioning and perceived effort, fatigue, and mood were taken during each stage of testing.

Results-Depressed patients performed worst on cognitive tests at baseline. During the treadmill test, patients with chronic fatigue syndrome had higher ratings of perceived effort and fatigue than both control groups, whereas patients with depression reported lower mood. After exertion, patients with chronic fatigue syndrome showed a greater decrease than healthy controls on everyday tests of focused $(p=0.02)$ and sustained $(p=0.001)$ attention, as well as greater deterioration than depressed patients on the focused attention task $(p=0.03)$. No between group differences were found in cardiovascular or symptom measures taken during the cognitive testing.

Conclusions-Patients with chronic fatigue syndrome show a specific sensitivity to the effects of exertion on effortful cognitive functioning. This occurs despite subjective and objective evidence of effort allocation in chronic fatigue syndrome, suggesting that patients have reduced working memory capacity, or a greater demand to monitor cognitive processes, or both. Further insight into the pathophysiology of the core complaints in chronic fatigue syndrome is likely to be realised by studying the effects of exercise on other aspects of everyday functioning.

(F Neurol Neurosurg Psychiatry 1998;65:541-546)
\end{abstract}

Keywords: chronic fatigue syndrome; cognition; exercise

, Telephone 0044

1315376671 ; fax 0044131

47 6860; email

S.Lawrie@ed.ac.uk

Received 8 August 1997 and in revised form

26 November 1997

Accepted 9 December 1997

Chronic fatigue syndrome is a complex condition characterised by fatigue on relatively trivial exertion, accompanied by various other com- plaints including myalgia, impaired memory and concentration, depressed mood, and anxiety. ${ }^{1}$ The aetiology and pathophysiology of the disorder remain uncertain, although there is some evidence for the importance of both infectious and psychosocial factors in causation..$^{2-4}$ Despite the subjective experience of muscle fatigue and pain, neurophysiological investigations have shown normal muscle strength and function in chronic fatigue syndrome; however, patients have reduced exercise tolerance and consistently report increased perception of effort at maximal and submaximal levels of exertion compared with healthy controls. ${ }^{5-8}$ This suggests CNS dysfunction, as do several reports of disturbed cognitive performance in chronic fatigue syndrome. ${ }^{9-13}$ The nature and extent of cognitive impairment is uncertain as memory, information processing, and attention may all be affected, but there is a gradually emerging consensus that patients with chronic fatigue syndrome may show particular deficits in effortful cognition. ${ }^{11-13}$ Further research is therefore necessary to examine the extent to which cognitive performance and effort may be linked to physical exertion in this syndrome.

We have recently proposed that chronic fatigue syndrome is best understood as a primary disturbance in the sense of effort accompanying physical and cognitive exertion. ${ }^{14}$ This hypothesis is in keeping with patient reports of symptom exacerbation after motor or mental activity and predicts that greater deficits will be apparent during the performance of controlled than during automatic tasks. However, as many patients with chronic fatigue syndrome also satisfy criteria for depressive disorders, ${ }^{4}$ and abnormalities on effortful cognition may also be found in depression, ${ }^{14}$ any test of this hypothesis must also include depressed patients. We therefore examined the muscular, cognitive, symptomatic, and effort perception responses to physical exertion in patents with chronic fatigue syndrome, patients with major depressive disorder, and healthy subjects. Our specific prediction was that patients with chronic fatigue syndrome would perform effortful cognitive tasks less well after exercise than the control groups, and that these deficits would be associated with increases in fatigue and effort perception.

\section{Methods}

We recruited 10 ambulant patients satisfying criteria for chronic fatigue syndrome ${ }^{1}$ from a 
Table 1 Test order

\begin{tabular}{|c|c|c|}
\hline Test phase & Measures recorded & Time (min) \\
\hline Baseline measures & Wt, $\mathrm{ETCO}_{2}, \mathrm{HR}, \mathrm{BP}, \mathrm{RPE}, \mathrm{PF}, \mathrm{MF}, \mathrm{NM}$ & 5 \\
\hline \multicolumn{3}{|l|}{ Initial test trial: } \\
\hline \multicolumn{3}{|l|}{ Isometric tests: } \\
\hline Grip strength & MVC $(\mathrm{kg})$ & \\
\hline $50 \% \mathrm{MVC}, 30 \mathrm{~s}$ & HR, BP, RPE, PF, MF, NM & 5 \\
\hline \multicolumn{3}{|l|}{ Cognitive tests: } \\
\hline Digit span & & 5 \\
\hline Digit symbol & & 2 \\
\hline Verbal fluency & & 2 \\
\hline Telephone search & & 4 \\
\hline Lottery & HR, BP, RPE, PF, MF, NM & 12 \\
\hline \multicolumn{3}{|l|}{ Treadmill test: } \\
\hline Set up: & & 5 \\
\hline Stage 1 & $\mathrm{ETCO}_{2}, \mathrm{HR}, \mathrm{BP}, \mathrm{RPE}, \mathrm{PF}, \mathrm{MF}, \mathrm{NM}$ & 3 \\
\hline Stage 2 & HR, BP, RPE, PF, MF, NM & 3 \\
\hline Stage 3 & $\mathrm{HR}, \mathrm{BP}, \mathrm{RPE}, \mathrm{PF}, \mathrm{MF}, \mathrm{NM}$ & 3 \\
\hline Stage 4 & HR, BP, RPE, PF, MF, NM & 3 \\
\hline Stage $5^{\star}$ & HR, BP, RPE, PF, MF, NM & 3 \\
\hline \multirow[t]{2}{*}{ Recovery } & $\mathrm{ETCO}_{2}$ & 2 \\
\hline & & 3 \\
\hline \multicolumn{3}{|l|}{ Repeat test trial: } \\
\hline Isometric tests: & & 5 \\
\hline Grip strength & MVC $(\mathrm{kg})$ & \\
\hline $50 \% \mathrm{MVC}, 30 \mathrm{~s}$ & HR, BP, RPE, PF, MF, NM & \\
\hline \multicolumn{3}{|l|}{ Cognitive tests: } \\
\hline Digit span & & 5 \\
\hline Digit symbol & & 2 \\
\hline Verbal fluency & & 2 \\
\hline Telephone search & & 4 \\
\hline \multirow[t]{2}{*}{ Lottery } & HR, BP, RPE, PF, MF, NM & 12 \\
\hline & Total time & 90 \\
\hline
\end{tabular}

*Test ended at $85 \%$ age predicted maximum heart rate ( $85 \%$ HRmax); stages completed and time taken varied between subjects. $\mathrm{HR}=$ Heart rate; $\mathrm{BP}=$ blood pressure; $\mathrm{RPE}=$ rating of perceived exertion; $\mathrm{PF}=$ physical fatigue $\mathrm{MF}=$ mental fatigue; $\mathrm{NM}=$ negative $\operatorname{mood} ; \mathrm{MVC}=$ maximal volun tary contraction; $\mathrm{ETCO}_{2}=$ end tidal $\mathrm{CO}_{2}$.

local infectious diseases clinic, 10 patients with unipolar major depressive disorder ${ }^{15}$ from a local psychiatric hospital, and 10 healthy controls from the local community. The subject groups were balanced for age (range 25-60) and sex, and screened for major physical or comorbid psychiatric disorders before participation. Patients with chronic fatigue syndrome and major depression were excluded. Ethical approval was obtained from the appropriate local health board committee.

During a preliminary meeting, subjects were assessed for levels of functioning on the SF-36, ${ }^{16}$ anxiety and depression on the hospital anxiety and depression (HAD) scale, ${ }^{17}$ premorbid intelligence, ${ }^{18}$ and severity of fatigue. ${ }^{19}$ Subjective ratings of physical fitness were measured on a five point scale ${ }^{20}$ and current levels of physical activity established according to the four categories (sedentary, irregular moderate, regular moderate, and regular vigorous) of the Health Education Authority. ${ }^{21}$

One to six days later, between 1230 and 530 pm, subjects performed a laboratory exercise test. The session included measures of hand grip strength and a battery of cognitive tests delivered at specific time intervals before and after vigorous isotonic exercise on a motorised treadmill (table 1). Heart rate, blood pressure, and rating of perceived exertion, ${ }^{22}$ along with 10 point ratings of physical fatigue, mental fatigue, and negative mood were taken after each block of neuropsychological and physical tasks. Physical fatigue was defined as a "sensation of tiredness or weakness in the muscles" and mental fatigue was "tiredness or fugginess of the brain or mind". The negative mood scale ranged from 0 (neutral) to 10 (extremely depressed) and subjects were asked to express elated mood (anything above neutral) as zero. To detect any hyperventilation at rest and two minutes after exercise, end tidal $\mathrm{CO}_{2}$ was measured with an end tidal analyser (Normocap 200, Datex, Finland) as the mean reading $(\mathrm{kPa})$ from 10 consecutive breaths.

Maximal voluntary contraction (MVC) was measured using a hand grip dynamometer (Jamar, JA Preston, USA). Subjects performed three trials, (3-5 s duration), using the dominant arm, and the mean score was recorded to the nearest $\mathrm{kg}$. Subjects were then asked to hold a sustained isometric contraction ( $50 \%$ of MVC for 30 seconds) during which measures of heart rate, blood pressure, ratings of perceived exertion, physical fatigue, mental fatigue, and negative mood were recorded.

Subjects then completed a series of neuropsychological tests. The following aspects of cognitive function were examined (in order): working memory/auditory attention (digit span, from WAIS-R, ${ }^{23}$ presented and scored as described by Randt and Brown ${ }^{24}$ ); psychomotor speed (digit symbol, also from WAIS-R); word fluency (FAS test, ${ }^{25}$ using the letters F and S only); and selective attention and sustained attention (telephone search and lottery tasks respectively, both from the test of everyday attention $\left.{ }^{26}\right)$. The telephone search is a timed task requiring subjects to visually scan a directory of telephone numbers and to mark entries labelled by target symbols. Scoring is determined by the number of correctly identified items, relative to the time taken, and scaled by age (range $0-19$ ). The lottery task requires subjects to listen to a prerecorded tape (of 10 minutes duration) containing a long series of letter and number combinations (two letters followed by a three digit number). Subjects are instructed to listen for numbers ending in 55 (88 in parallel version) and to write down the letters immediately preceding that number. One point is scored for each response where at least one letter is correct and in the correct position (age scaled, range $0-13$ ).

Subjects then performed a symptom limited progressive treadmill exercise test using the standard Bruce protocol, ${ }^{27}$ modified by the inclusion of an initial stage at a $5 \%$ gradient (after Riley et $\mathrm{al}^{6}$ ). Measures of heart rate, blood pressure, rating of perceived exertion, physical fatigue, mental fatigue, and negative mood were were recorded during the last 45 seconds of each 3 minute stage. Heart rate was monitored throughout by 10 lead ECG (Marquette Electronics Inc, USA). To standardise the level of physiological demand, all subjects were exercised to a target of $85 \%$ age predicted maximum heart rate $(85 \%$ HRmax; $220-$ age in years). This level was selected as being sufficiently vigorous to place some physiological stress on the subject yet low enough to be achieved by those with chronic fatigue syndrome.

The second battery of strength tests and parallel versions of the neuropsychological tests were started after five minutes of recovery 
Table 2 Clinical characteristics of subject groups (mean (SD))

\begin{tabular}{llll}
\hline & Controls $(n=10)$ & CFS $(n=10)$ & MDD $(n=10)$ \\
\hline Age (y) & $40.5(10.4)$ & $40.5(9.1)$ & $38.1(10.6)$ \\
Sex ratio (F/M) & $7 / 3$ & $7 / 3$ & $7 / 3$ \\
Weight (kg) & $73.7(18.8)$ & $68.0(14.1)$ & $70.7(13.0)$ \\
NART (full scale IQ) & $113.5(11.1)$ & $116.0(8.9)$ & $115.6(7.0)$ \\
Duration of illness (y) & N/A & $7.1(4.8)$ & $5.1(6.9)$ \\
Chalder fatigue score & N/A & $27.9(4.0)$ & $14.8(6.9)$ \\
End tidal CO at rest (kPa) & $4.8(0.5)$ & $4.8(0.4)$ & $4.4(0.5)$ \\
HAD (anxiety) & & & \\
HAD (depression) & $3.6(1.8)$ & $5.5(1.6)$ & $11.4(4.8)$ \\
HAD f* (excluding Q4) & $0.6(0.7)$ & $6.1(2.0)$ & $9.1(5.8)$ \\
& $0.2(0.4)$ & $3.5(1.7)$ & $8.2(5.7)$ \\
SF36 (scaled scores, (\%)): & & & \\
Physical functioning & & & \\
Mental health & $96.5(2.4)$ & $41.5(21.7)$ & $81.0(18.1)$ \\
Role physical & $85.6(7.8)$ & $70.8(9.6)$ & $46.4(24.5)$ \\
Bodily pain & $100.0(0.0)$ & $20.0(36.9)$ & $45.0(45.3)$ \\
Role emotional & $92.8(12.0)$ & $54.0(27.1)$ & $74.1(21.7)$ \\
General health & $96.7(10.5)$ & $86.6(28.3)$ & $30.0(39.9)$ \\
Social functioning & $84.4(17.6)$ & $33.2(14.7)$ & $45.8(20.5)$ \\
Vitality & $96.3(8.4)$ & $41.3(31.3)$ & $45.0(32.4)$ \\
\hline
\end{tabular}

^HAD-f refers to depression subscale score excluding Q4 on fatigue.

Table 3 Baseline isometric grip strength and cognitive performance (mean (SD))

\begin{tabular}{lrrrl}
\hline & Controls & \multicolumn{1}{c}{ CFS } & MDD & $\begin{array}{l}\text { Kruskal-Wallis } \\
\text { ANOVA, } p \text { value }\end{array}$ \\
\hline $\begin{array}{l}\text { Isometric grip strength MVC (kg) } \\
\text { Cognitive tests (scaled scores) }\end{array}$ & $35.5(11.4)$ & $33.1(8.5)$ & $35.0(7.8)$ & $\chi^{2}=0.2 \mathrm{p}=0.9$ \\
Digit span (forwards) & $7.5(1.4)$ & $7.1(1.2)$ & $7.4(1.4)$ & $\chi^{2}=0.7, \mathrm{p}=0.7$ \\
Digit span (backwards) & $6.1(1.7)$ & $6.1(1.2)$ & $4.8(1.1)$ & $\chi^{2}=6.5, \mathrm{p}=0.04$ \\
Digit symbol substitution & $13.4(2.6)$ & $12.3(2.5)$ & $11.9(2.0)$ & $\chi^{2}=1.8, \mathrm{p}=0.4$ \\
Word fluency (letter S only) & $19.7(7.0)$ & $18.7(5.1)$ & $18.8(5.7)$ & $\chi^{2}=0.0, \mathrm{p}=1.0$ \\
Telephone search & $11.8(3.2)$ & $11.2(2.9)$ & $10.1(3.4)$ & $\chi^{2}=0.8, \mathrm{p}=0.7$ \\
Lottery & $10.7(3.1)$ & $10.4(2.6)$ & $9.6(3.2)$ & $\chi^{2}=1.9, \mathrm{p}=0.4$ \\
\hline
\end{tabular}

time. Repeat tests were administered in the same order and following the same procedures as before.

STATISTICAL ANALYSIS

Differences between the groups in treadmill test cardiovascular indices (at rest and 85\% HRmax) were determined by one way analysis of variance (ANOVA). Baseline and postexer- tion changes in performance on the cognitive and isometric strength tests were examined for between group differences using the KruskalWallis one way ANOVA for non-parametric data. Baseline and changes in subjective ratings of effort, fatigue, and mood were also assessed using this test. Where appropriate, post hoc analysis was conducted using the MannWhitney $U$ test. The value of $\alpha$ was set at 0.05 .

\section{Results}

Table 2 shows that subjects were well matched for age, sex ratio, weight, and IQ. The mean duration of illness was similar for the major depressive disorder and chronic fatigue syndrome groups, with both showing wide variation between individual patients (range 1-23 years and 3-16 years respectively). All patients with major depressive disorder and three patients with chronic fatigue syndrome were taking antidepressants at the time of testing, with dosage stable for at least two weeks before participation. As expected, patients with major depressive disorder scored substantially higher on the HAD subscales than the chronic fatigue syndrome and control groups (all overall $\mathrm{p}$ values $<0.001)$. Patients with chronic fatigue syndrome were more depressed and more anxious than controls but none reached criterion levels (11 or more) for comorbid anxiety or depression. Substantial between group differences were also found on all items of the SF36. Activity levels also showed some variation between groups. More control subjects participated in regular vigorous physical activity (controls 20\%; major depressive disorder 10\%; chronic fatigue syndrome $0 \%$ ) and regular moderate exercise (controls 50\%; major depressive disorder $50 \%$; chronic fatigue syndrome 10\%). Most patients with chronic

Table 4 Cardiovascular measures and subjective ratings during treadmill exercise (mean (SD) or median (range))

\begin{tabular}{|c|c|c|c|c|c|}
\hline & Rest & Stage 1 & Stage 2 & Stage 3 & $85 \%$ HRmax \\
\hline \multicolumn{6}{|c|}{ Mean heart rate $\left(\right.$ b. $\left.\min ^{-1}\right)$ : } \\
\hline Controls & $68.6(6.9)$ & $105.7(7.3)$ & $116.5(7.2)$ & $139.4(9.6)$ & $157.6(11.6)$ \\
\hline CFS & $74.9(7.8)$ & $108.1(16.2)$ & $117.4(10.5)$ & $136.4(12.1)$ & $151.1(19.8)^{5}$ \\
\hline MDD & $81.2(15.5) \dagger$ & $121.6(18.0)$ & $132.0(19.0)$ & $142.6(11.7)$ & $157.7(8.3)$ \\
\hline \multicolumn{6}{|c|}{ Mean systolic blood pressure $(\mathrm{mm} \mathrm{Hg})$ : } \\
\hline Controls & $118.6(15.3)$ & $136.3(13.6)$ & $146.8(20.7)$ & $158.0(27.7)$ & $154.9(25.2)$ \\
\hline CFS & $110.2(8.3)$ & $131.8(13.4)$ & $143.0(13.7)$ & $141.0(14.7)$ & $148.4(13.3)$ \\
\hline MDD & $114.2(16.2)$ & $130.9(23.4)$ & $142.3(17.7)$ & $147.5(1.3)$ & $143.3(22.5)$ \\
\hline \multicolumn{6}{|c|}{ Mean diastolic blood pressure (mm Hg): } \\
\hline Controls & $79.8(10.7)$ & $84.7(11.3)$ & $83.8(13.6)$ & $86.0(12.3)$ & $87.3(10.9)$ \\
\hline CFS & $76.4(9.5)$ & $85.8(9.2)$ & $85.8(8.8)$ & $78.5(8.4)$ & $88.2(8.6)$ \\
\hline MDD & $80.6(12.6)$ & $82.0(12.0)$ & $84.3(10.9)$ & $78.0(2.8)$ & $83.8(11.2)$ \\
\hline \multicolumn{6}{|c|}{ Median rating of perceived exertion: } \\
\hline Controls & $0 \quad(0-1)$ & $3(1-3)$ & $3(1-4)$ & $3.5(1-4)$ & $4 \quad(3-6)$ \\
\hline CFS & $1 \quad(0-2)$ & $3.5(2-10)$ & $4 \quad(3-7)$ & $5 \quad(4-10)$ & $8 \quad(5-10)^{\star \star} \neq \ddagger$ \\
\hline MDD & $0 \quad(0-4)$ & $2.5(2-7)$ & $4 \quad(2-7)$ & $5 \quad(3-6)$ & $5 \quad(4-7) \dagger$ \\
\hline \multicolumn{6}{|c|}{ Median rating of physical fatigue: } \\
\hline Controls & $0 \quad(0-2)$ & $1.5(0-4)$ & $2(0-4)$ & $2.5(1-5)$ & $3.5(2-6)$ \\
\hline CFS & $2.5(0-4)^{\star \star}$ & $4.5(2-9)$ & $6 \quad(4-7)$ & $6 \quad(5-9)$ & $8 \quad(6-9)^{\star \star \star ⿰ 扌 \ddagger ~}$ \\
\hline MDD & $0.5(0-6)$ & $3.5(0-7)$ & $5 \quad(0-7)$ & $5 \quad(4-7)$ & $6 \quad(4-8)+\dagger$ \\
\hline \multicolumn{6}{|c|}{ Median rating of mental fatigue: } \\
\hline Controls & $0 \quad(0-2)$ & $0 \quad(0-2)$ & $0 \quad(0-2)$ & $0 \quad(0-3)$ & $0 \quad(0-3)$ \\
\hline CFS & $2.5(0-5)^{\star \star}$ & $4 \quad(1-5)$ & $4 \quad(1-6)$ & $4 \quad(1-6)$ & $4 \quad(1-6)^{\star \star} \ddagger$ \\
\hline MDD & $3 \quad(0-5) \dagger$ & $1.5(0-6)$ & $3(0-7)$ & $4 \quad(0-5)$ & $3(0-5)$ \\
\hline \multicolumn{6}{|c|}{ Median rating of mood: } \\
\hline Controls & $0 \quad(0-2)$ & $0 \quad(0-1)$ & $0 \quad(0-2)$ & $0 \quad(0-3)$ & $0 \quad(0-3)$ \\
\hline CFS & $0 \quad(0-2)$ & $0.5(0-3)$ & $2(0-3)$ & $2 \quad(0-4)$ & $2(0-4)$ \\
\hline MDD & $2.5(0-7) \dagger \ddagger$ & $3(0-6)$ & $3.5(0-7)$ & $4 \quad(0-6)$ & $2.5(0-6) \dagger$ \\
\hline
\end{tabular}

${ }^{\star} \mathrm{p}<0.05,{ }^{\star \star} \mathrm{p}<0.01$ chronic fatigue syndrome $v$ controls.

$\mathrm{tp}<0.05,+\mathrm{t}<<0.01$ major depressive disorder $v$ controls.

$\neq \mathrm{p}<0.01, \neq \neq \mathrm{p}<0.01$ chronic fatigue syndrome $v$ major depressive disorder.

§Two subjects with CFS did not reach $85 \%$ maximum heart rate. When removed from analysis mean heart rate for the CFS group is $158.5(\mathrm{SD} \mathrm{12.1})$ b. $\mathrm{min}^{-1}$. 
Table 5 Median (range) changes in cognitive test performance, cardiovascular indices, and subjective scores from assessments before to after exercise

\begin{tabular}{lcccll}
\hline & Controls & CFS & MDD & Kruskal Wallis & Final test \\
\hline Digit span (forwards) & $0.0(-2$ to 1$)$ & $-0.5(-2$ to 2$)$ & $0.0(-2$ to 0$)$ & $\chi^{2}=1.2, \mathrm{p}=0.6$ \\
Digit span (backwards) & $0.0(-2$ to 2$)$ & $0.0(-2$ to 2$)$ & $0.0(-1$ to 2$)$ & $\chi^{2}=0.4, \mathrm{p}=0.8$ \\
Digit symbol substitution & $2.5(-6$ to 8$)$ & $0.0(-2$ to 3$)$ & $1.0(0$ to 2$)$ & $\chi^{2}=3.2, \mathrm{p}=0.2$ \\
Word fluency & $-3.5(-15$ to 3$)$ & $-2.0(-9$ to 2$)$ & $-3.5(-10$ to 2$)$ & $\chi^{2}=0.01, \mathrm{p}=0.9$ & \\
Telephone search & $-1.0(-6$ to 1$)$ & $-4.0(-7$ to -1$)$ & $-1.0(-6$ to 1$)$ & $\chi^{2}=7.2, \mathrm{p}=0.03$ & CFS $>$ MDD, $\mathrm{p}=0.03$ \\
& & & & & \\
Lottery & $0.0(-1$ to 7$)$ & $-4.5(-9$ to 0$)$ & $-1 \quad(-8$ to 4$)$ & $\chi^{2}=11.0, \mathrm{p}=0.004$ & CFS $>$ CON, $\mathrm{p}=0.02$ \\
Change in heart rate & $6.0(-6$ to 18$)$ & $1.5(-6$ to 10$)$ & $4 \quad(0$ to 12$)$ & $\chi^{2}=0.9, \mathrm{p}=0.6$ & \\
Change in systolic BP & $-1.0(-14$ to 10$)$ & $1.0(-20$ to 8$)$ & $3 \quad(-6$ to 10$)$ & $\chi^{2}=1.8, \mathrm{p}=0.4$ & \\
Change in diastolic BP & $-3.5(-12$ to 10$)$ & $-1.0(-8$ to 10$)$ & $1.0(-6$ to 10$)$ & $\chi^{2}=2.1, \mathrm{p}=0.4$ & \\
Change in perceived effort & $0.0(-3$ to 1$)$ & $1.5(-3$ to 3$)$ & $-0.5(-3$ to 2$)$ & $\chi^{2}=4.2, \mathrm{p}=0.1$ \\
Change in physical fatigue & $0.0(-2$ to 3$)$ & $1.0(-3$ to 5$)$ & $2.0(-6$ to 6$)$ & $\chi^{2}=4.2, \mathrm{p}=0.1$ \\
Change in mental fatigue & $0.5(-3$ to 2$)$ & $1 \quad(-5$ to 3$)$ & $0.5(-4$ to 4$)$ & $\chi^{2}=1.1, \mathrm{p}=0.6$ & \\
Change in mood & $0.0(-4$ to 0$)$ & $0.0(-2$ to 5$)$ & $0.5(-4$ to 3$)$ & $\chi^{2}=4.4 \mathrm{p}=0.1$ \\
\hline
\end{tabular}

Negative values in cognitive and cardiovascular scores indicate a decline in test performance from trial 1 to trial 2. Negative values in subjective ratings indicate a drop in perceived rating of effort, fatigue, or negative mood - that is, improved subjective experience. $\mathrm{CFS}=$ chronic fatigue syndrome; $\mathrm{MDD}=$ major depression disorder; $\mathrm{CON}=$ controls

fatigue syndrome undertook occasional moderate exercise (controls 30\%; major depressive disorder $40 \%$; chronic fatigue syndrome $50 \%$ ) but four were extremely sedentary $(<30$ minutes moderate exercise a week), (controls $0 \%$, major depressive disorder $0 \%$; chronic fatigue syndrome $40 \%$ ). Many patients with chronic fatigue syndrome and patients with major depressive disorder perceived themselves to be of low physical fitness $(80 \%$ of patients with chronic fatigue syndrome and $50 \%$ with major depressive disorder rated themselves as "below average" compared with $20 \%$ of controls).

At baseline cognitive testing, depressed patients had significantly lower scores on the backwards digit span test compared with the chronic fatigue syndrome group $(z=2.55$, $p=0.01$ ), whereas the scores on all other cognitive tests were similar between the three groups (table 3). No group differences in baseline maximal voluntary contraction were seen.

Eight of the patients with chronic fatigue syndrome $(80 \%)$ and all of the healthy and depressed groups achieved the target $85 \%$ HRmax. Measures of end tidal $\mathrm{CO}_{2}$ showed that only one patient, with major depressive disorder, hyperventilated during the test $(<4.0$ $\mathrm{kPa}$ ). Table 4 shows that during the initial stages of the treadmill test, patients with major depressive disorder had higher mean heart rate; but at $85 \% \mathrm{HRmax}$, mean heart rate and blood pressure were similar for all three groups. At rest, rating of physical fatigue and mental fatigue was higher in the chronic fatigue group compared with normal controls $(z=3.18$, $\mathrm{p}=0.002$ and $\mathrm{z}=2.85, \mathrm{p}=0.004$ respectively), whereas negative mood was significantly higher in the depressed group than both chronic fatigue syndrome and control groups $(\mathrm{z}=2.45$, $\mathrm{p}=0.015 ; \mathrm{z}=2.14, \mathrm{p}=0.03)$. At $85 \% \mathrm{HRmax}$, patients with chronic fatigue syndrome reported significantly greater perceived exertion, greater physical fatigue, and greater mental fatigue than controls and patients with major depressive disorder. The major depressive disorder group indicated significantly greater negative mood than controls $(\mathrm{z}=2.09, \mathrm{p}=0.04)$, but the level indicated was unchanged from baseline ratings.

As predicted, the chronic fatigue group showed a significantly greater decline in per- formance after exercise on the telephone search and lottery tasks than the controls (table 5). Performance on the telephone search task also declined significantly compared with the major depressive disorder group. This change in performance was not accompanied by any significant change in cardiovascular indices, perceived exertion, physical fatigue, mental fatigue, or negative mood after the treadmill test. On the isometric strength task, before and after exercise, the three groups showed no differences in maximal voluntary contraction or in the heart rate and blood pressure measures during the $50 \%$ MVC sustained contraction. Subjective ratings, however, did show significant group differences $\left(\chi^{2}=6.1 \mathrm{p}=0.048\right.$ and $\chi^{2}=7.4, p=0.02$ respectively) with the chronic fatigue syndrome group reporting greater increases in mental fatigue and negative mood than the control group $(p=0.005$ and $p=0.01$ respectively).

\section{Discussion}

Our main finding is that patients with chronic fatigue syndrome show a specific response to exercise, characterised by an increased perception of effort and deficits in postexertional cognitive processing. We are not aware of any previous studies which have examined both motor and cognitive domains experimentally. Patients with chronic fatigue syndrome often report delayed physical and mental fatigue (for up to four days) after exertion, but this remains controversial experimentally ${ }^{78}$ and we have shown that some deficits in cognitive performance may be found within half an hour of strenuous exercise. The fact that we found specific decrements in cognitive performance after exercise despite no between group differences in the accompanying objective cardiovascular indices argues against any simple lack of motivation on the part of patients with chronic fatigue syndrome. Our results are in keeping with our hypothesis ${ }^{14}$ that the most distinctive subjective complaint in chronic fatigue syndrome is of increased effort and the most distinctive objective impairment is in effort processing-both being most evident after exercise.

We employed a relatively small subject sample to examine responses to exertion, but the groups were well matched and the power of the study was sufficient to identify clear differences 
between the chronic fatigue syndrome and control groups. The differences cannot be attributed to simple effects of exhaustion as the control groups did not show them and they were only evident on some of the tests. Depression or anxiety are also unlikely causes of the effects of exercise as our patients with chronic fatigue syndrome scored substantially lower on these subscales of the HAD than those with depression. Similarly, habitual activity levels were reduced in both the chronic fatigue syndrome and depressed groups.

At baseline, the only between group difference was the deficit on short term memory in major depressive disorder. The clear cut differences in chronic fatigue syndrome after exertion highlight the general importance of using appropriate challenge tests in attempting to differentiate particular disorders. The treadmill test we employed may be a generally useful provocation measure in chronic fatigue syndrome. The fact that we found differences between the diagnostic groups despite two patients with chronic fatigue syndrome not being able to tolerate $85 \%$ HRmax only strengthens our findings overall. Our results confirm earlier reports that patients with chronic fatigue syndrome do not show grossly abnormal cardiovascular responses to exercise but do have a markedly increased perception of effort at equivalent workloads. ${ }^{68}$ We have also shown higher physical and mental fatigue on exertion in chronic fatigue syndrome, as compared with depressed and normal controls, whereas significant changes in major depressive disorder were confined to lower mood. Chronic fatigue syndrome and major depressive disorder can therefore be differentiated by their response to exercise as well as in terms of their primary complaints.

Cognitive testing after exercise showed the greatest deficits in patients with chronic fatigue syndrome on the effortful tests of attention (telephone search and lottery). These results are in line with previous reports ${ }^{11}$ and show the predicted differential sensitivity to exercise in chronic fatigue syndrome. In particular, the large deficit in telephone search performance in those with chronic fatigue syndrome-of four points on a 19 point scale-differentiated them from both major depressive disorder and healthy controls. This striking result accords with clinical reports because we examined the effects of exertion on an everyday test of focused attention ${ }^{26}$ - an experimental situation that approximates to the usual experience of patients. The similar pattern of results on the lottery test - a more difficult and longer test of selective attention-and the change in the perception of effort on mental exertion may have been significant had we studied a larger sample.

No group differences in muscle strength were identified. Given that the reported levels of habitual activity varied between groups (controls most active, chronic fatigue syndrome least active) this is surprising but supports earlier research findings. ${ }^{57}$ This suggests that patients with chronic fatigue syndrome retain their capacity for short duration isometric tasks, despite finding them more mentally taxing.

Our results have clear implications for our effort hypothesis ${ }^{14}$ and further research into the pathophysiology and treatment of chronic fatigue syndrome. The experience of increased effort supports our view that patients with chronic fatigue syndrome may have a reduced working memory capacity or a greater operational need to monitor cognitive (and muscular) exertion, demanding a relatively greater increase in effort for a given task. In other words, automatic processes become controlled and effortful. It is striking that this seems to overlap for motor and cognitive domains in chronic fatigue syndrome. Successful treatment may therefore depend on regaining automaticity in cognitive and motor function, which could be achieved by either a graded return to such activities or by explicitly modifying feedback during performance. It is of interest that graded exercise is the only common component of the only treatments of established benefit in chronic fatigue syndrome. ${ }^{28-30}$

In summary, we have shown abnormalities on effortful neuropsychological tasks after exertion that differentiate those with chronic fatigue syndrome from those with major depressive disorder or healthy controls. The increases in perceived effort and subjective fatigue in chronic fatigue syndrome support their role in discriminating the disorders. Furthermore, exercise induced changes in performance, fatigue, and effort are a more sensitive measure of function in chronic fatigue syndrome than are resting measures.

We are grateful to the Scottish Office Home and Health Department for funding, to Dr Ian Todd for access to the exercise treadmill, to Ms Marjorie Bennet for conducting the treadmill tests, to Dr Ronan O'Carroll for advice on cognitive testing, and to Dr Michael Sharpe for comments on a draft of this article. Finally, we thank all the subjects for their assistance.

1 Fukuda K, Straus SE, Hickie I, et al. The chronic fatigue syndrome: approach to its definition and study. Ann Intern syndrome: approach to

2 Cope H, David A, Pelosi A, et al. Predictors of chronic postviral fatigue. Lancet 1994;344:864-8.

3 Wessely S, Chalder T, Hirsch S, et al. Postinfectious fatigue: prospective cohort study in primary care. Lancet 1995;345: $1333-8$

4 Lawrie SM, Manders DN, Geddes JR, et al. A populationbased incidence study of chronic fatigue. Psychol Med 1997;27:343-53.

5 Stokes MJ, Cooper RG, Edwards RHT. Normal muscle strength and fatiguability in patients with effort syndromes. $B M \mathcal{F}$ 1988;297:1014-7.

6 Riley MS, O'Brien CJ, McCluskey DR, et al. Aerobic work capacity in patients with chronic fatigue syndrome. BMF 1990;301:953-6.

7 Gibson H, Carroll N, Clague JE, et al. Exercise performance and fatiguability in patients with chronic fatigue syndrome. and fatiguability in patients with chronic fatigue

8 Sisto SA, LaManca J, Cordero DL, et al. Metabolic and cardiovascular effects of a progressive exercise test on patients with chronic fatigue syndrome. Am $7 \mathrm{Med}$ 1996;100:634-40.

9 McDonald E, Cope H, David A. Cognitive impairment in patients with chronic fatigue: a preliminary study. $\mathcal{F}$ Neurol Neurosurg Psychiatry 1993;56:812-5.

10 Ray C, Phillips L, Weir WRC. Quality of attention in chronic fatigue syndrome: subjective reports of everyday ttention and cognitive difficulty, and performs of focused attention. Br f Clin Psychol 1993;32:357-64.

11 DeLuca J, Johnson SK, Beldowitz D, et al. Neuropsychological impairments in chronic fatigue syndrome, multiple sclerosis, and depression. I Neurol Neurosurg Psychiatry 1995;58:38-43.

12 Joyce E, Blumenthal S, Wessely S. Memory, attention and executive function in chronic fatigue syndrome. 7 Neurol Neurosurg Psychiatry 1996;60:495-503. 
13 Wearden A, Appleby L. Cognitive performance and complaints of cognitive impairment in chronic fatigue syncomplaints of cognitive impairment
drome. Psychol Med 1997;27:81-90.

14 Lawrie SM, MacHale SM, Power MJ, et al. Hypothesis: is the chronic fatigue syndrome best understood as a primary disturbance in the sense of effort? Psychol Med 1997;27 995-9.

15 American Psychiatric Association. Diagnostic and statistical manual of mental disorders, 4th ed. Washington, DC: APA, 1994.

16 Ware JE, Sherbourne CD. The medical outcomes study 36-item short form health survey (SF-36): I. Conceptual
framework and item selection. Med Care 1992;30:473-83.

17 Zigmond AS, Snaith RP. The hospital anxiety and depression scale. Acta Psychiatr Scand 1983;67:361-70.

18 Nelson H, Willison J. National adult reading test. 2nd ed. Windsor: NFER-Nelson, 1991.

19 Chalder T, Berelowitz G, Pawlikowska T, et al. Development of a fatigue scale. F Psychosom Res 1993;37:147-53.

20 Forth CD, Salmoni AW. Relationships among self reported physical activity, aerobic fitness, and reaction time. Can 7 physical activity, aerobic fitn

21 Health Education Authority. Moving on: international perspectives on promoting physical activity. London: HEA, 1995.

22 Borg GAV Psychophysical basis of perceived exertion. Med Sci Sports Exerc 1982;14:377-81.

23 Wechsler D. Manual for the Wechsler adult intelligence scale revised. New York: Psychological Corporation, 1981.

24 Randt CT, Brown ER. Randt memory test. New York: Life Sciences Associates, 1983.

25 Benton AL, Hamsher K. Multilingual asphasia examination. Iowa: University of Iowa Hospitals, 1978.

26 Robertson IH, Ward T, Ridgeway V, et al. The test of everyday attention. England: Thames Valley Test Company, 1994.

27 Bruce RA. Exercise testing of patients with coronary artery disease. Ann Clin Res 1971;3:323-32.

28 Sharpe MC, Hawton K, Simkin S, et al. Cognitive behaviour therapy for the chronic fatigue syndrome: a randomised controlled trial. BMF 1996;312:22-6.

29 Deale A, Chalder T, Marks I, et al. Cognitive behaviour therapy for the chronic fatigue syndrome: a randomised controlled trial. Am f Psychiatry 1997;154:408-14.

30 Fulcher KY, White PD. Randomised controlled trial of graded exercise in patients with the chronic fatigue syndrome. $B M F$ 1997;314:1647-52 